\title{
Natural compounds for controlling Drosophila suzukii. A review
}

\author{
Doriane Dam ${ }^{1} \cdot$ Daniel Molitor $^{1} \cdot$ Marco Beyer $^{1}$ (D) \\ Accepted: 23 August 2019 / Published online: 13 November 2019 \\ (C) INRA and Springer-Verlag France SAS, part of Springer Nature 2019
}

\begin{abstract}
The drosophilid fly Drosophila suzukii is an invasive pest that has recently started threatening fruit production in Europe. In contrast to many other fruit flies, D. suzukii is able to lay eggs in ripening and mature fruits where larvae develop, rendering fruits unmarketable. This preference for ripening fruit requires pest control shortly before harvest, implying a high risk of residues on the fruit if synthetic insecticides are used. As the current management practices largely rely on chemical control, the need for alternative solutions has emerged. Here, we review the studies published up to now on the efficacy of natural compounds against D. suzukii. Several natural compounds were identified that act as repellents, contact or ingestion toxicants, fumigants, ovicides or oviposition deterrents. The most promising compounds of each group were (i) essential oils (EOs) such as the EO of thyme or its major ingredient thymol which repelled flies from fresh fruits for at least $24 \mathrm{~h}$; (ii) Leptospermum ericoides and L. scoparium EOs, which expressed contact toxicity at a $\mathrm{LD}_{50}<1.2 \mu \mathrm{g} / \mathrm{fly}$; (iii) the combination of erythritol and sucrose, which was a potent ingestion toxicant against adults and (iv) a chitinase from Euphorbia characias against larvae (both of the latter two resulted in $100 \%$ mortality); (v) the EO ingredients perilla aldehyde, geranial and neral showed the highest insecticidal activities as fumigants $\left(\mathrm{LC}_{50}<1.52 \mathrm{mg} / \mathrm{l}\right.$ air for males and $2.6 \mathrm{mg} / \mathrm{l}$ air for females) and (vi) powdered sulphur was reported to be the most efficient oviposition deterrent, reducing the number of eggs deposited into the fruits by $76 \%$. To enable a wider use of the natural compounds in sustainable agriculture, more information on (i) potential effects on non-target organisms, (ii) field performance and (iii) life cycle analyses results is currently needed.
\end{abstract}

Keywords Climate change $\cdot$ Crop protection $\cdot$ Insecticides $\cdot$ Reducing pesticides $\cdot$ Spotted wing drosophila

\begin{tabular}{ll}
\multicolumn{2}{l}{ Abbreviations } \\
AChE & Acetylcholinesterase \\
AST & Average survival time \\
BA & Butyl anthranilate \\
CT & Concentration tested \\
DEET & $N, N$-diethyl-meta-toluamide \\
DMB & Methyl 2,4-dimethoxy-6-methylbenzoate \\
EA & Ethyl anthranilate \\
EO & Essential oil \\
ESE & Ethanolic seed extract \\
GST & Glutathione-S-transferase \\
MDA & Methyl $N, N$-dimethylanthranilate
\end{tabular}

Marco Beyer marco.beyer@list.lu

1 Environmental Research and Innovation Department, Luxembourg Institute of Science and Technology, 41 Rue du Brill, 4422 Belvaux, Luxembourg

\section{Contents}

1. Introduction

1.1 Classification of tested compounds

1.2 General considerations on estimated toxicity parameters

2. Repellents

$2.1 \mathrm{~T}$ - maze and choice trap assays

2.2 Impregnated cotton wicks

2.3 Direct airborne repellent assay

2.4 Conclusion on repellents

3. Contact toxicants

3.1 Topical application

3.2 Use of impregnated matrices

3.3 Conclusion on contact toxicants

4. Ingestion or contact toxicants

4.1 Feeding and development on a supplemented medium

4.2 Feeding on and contact with a soaked cotton wick

4.3 Feeding on or contact with treated fruits

4.4 Conclusion on ingestion or contact toxicants

5. Fumigants 
6. Fumigants and contact toxicants

7. Oviposition repellents and ovicides

7.1 Raspberries

7.2 Strawberries

7.3 Blackberries

7.4 Blueberries

7.5 Conclusion concerning the effect on oviposition

8. Limitations of using natural compounds for controlling

D. suzukii

8.1 High concentrations needed for efficacy

8.2 Transfer to and deposition of natural compounds on crops

8.3 Competition for use

8.4 Occasional lack of specificity

8.5 Lack of knowledge of the (molecular) modes of action

8.6 Waiting time until effects unfold

Acknowledgements

Conflict of interest

References

\section{Introduction}

Drosophila suzukii (Matsumura), also called spotted wing drosophila, is a fruit fly with a clear sexual dimorphism: males have a dark spot on the wings while females have not (Fig. 1) (Cini et al. 2012). Native to Southeast Asia, D. suzukii was first observed in the USA as well as in Europe (Spain and Italy) in 2008. Since then, it has been spreading north- and eastwards (Asplen et al. 2015). In a climate change impact study, a temperature-driven population dynamics model using data derived from several Global Circulation Models (CMIP5) suggested that northern latitudes may experience increased D. suzukii populations due to milder winter conditions (Langille et al. 2017). In fact, D. suzukii reached

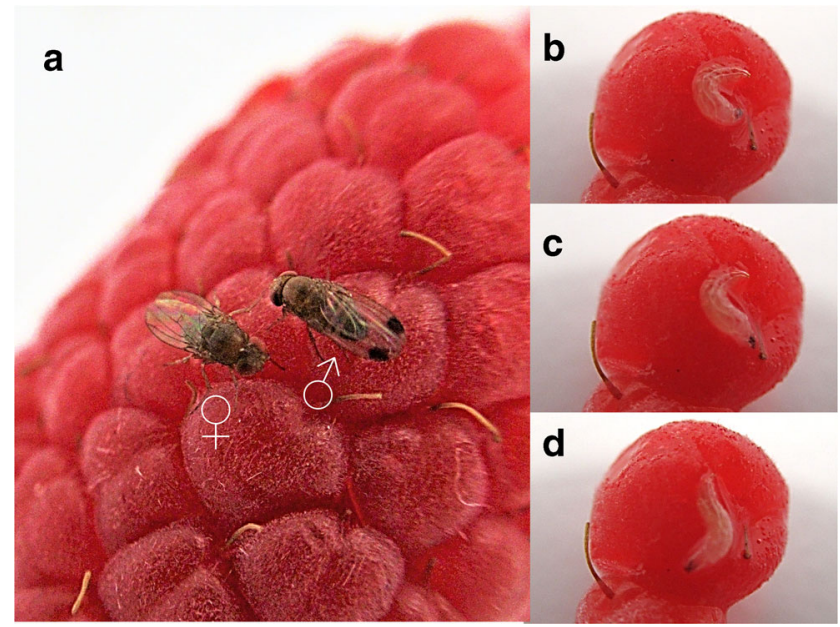

Fig. 1 a Female (left) and male (right, with spotted wings) adults of Drosophila suzukii on a raspberry fruit. b-d Time series (b $0 \mathrm{~s}, \mathbf{c} 45 \mathrm{~s}$, d 100 s) of a $D$. suzukii larvae feeding on a raspberry fruit
Luxembourg in 2014, where it was first observed on red wine cultivars after a very mild winter (Schultz and Molitor 2016). D. suzukii is considered as a serious pest for soft-skinned fruits for several reasons. First, their serrated ovipositor allows the females to pierce the skin of ripening and ripe fruit to deposit eggs inside of the fruit. After a few days, the eggs hatch and the larvae develop while feeding on the fruit tissue, rendering the infested fruit useless for direct sale and processing. The oviposition wound in the fruit skin allows infection by fungi and bacteria and increases the risk of rapid fruit decay. After the larval stage, pupation occurs and a new generation of adults emerges. Second, D. suzukii can use a wide range of wild and cultivated host plants, such as sweet cherry, strawberry, raspberry, apricot, plum, fig or grape for oviposition (Cini et al. 2012). Third, D. suzukii has a high fecundity coupled with a short generation cycle. Females can lay more than 400 eggs in their lifetime and at optimum conditions of temperature, humidity and food resources, 8 days is sufficient for an egg to become an adult fly. Multiple generations and an exponential increase of the population can easily occur during the cropping season (Cini et al. 2012; Hamby et al. 2016). Current management practices are based mainly on the application of synthetic insecticides (belonging to the families of the pyrethroids or organophosphates or the active ingredient spinetoram) or bioinsecticides (spinosad and pyrethrin). Between one and 8 applications are necessary to insure an effective protection during fruit ripening depending on the crop and its susceptibility, the pest pressure and other environmental factors (Asplen et al. 2015; Shawer et al. 2018). The chemical protection is often complemented by cultural measures including sanitation (leaf removal in the cluster zone, selective removal of ripe and overripe fruit from the parcels, covering the crops with nets) and the reduction of the harvest intervals (Asplen et al. 2015; Haye et al. 2016; Schultz and Molitor 2016). The biological control of D. suzukii is still under investigation. Some efficient parasitoids have been identified with most of them being native to Asia, while the predators identified so far had an insufficient effect on the D. suzukii population (Asplen et al. 2015; Haye et al. 2016). Concerning entomopathogenic nematodes and fungi, laboratory tests indicated good efficacy, but optimization with regard to survival during transport and in natural matrices like soil is needed before high control efficacy can be expected in the field (Asplen et al. 2015; Haye et al. 2016). Since D. suzukii has a strong preference for ripe fruit, little time remains for pesticide degradation before harvest and consumption if synthetic insecticides are used. Therefore, there is a strong interest in controlling D. suzukii by methods other than synthetic pesticides.

The purpose of this review was to identify the most effective natural extracts and compounds tested against $D$. suzukii up to now. We used the broad definition of natural compounds including biotic materials produced by organisms, and other 
natural materials, such as soil components or coal. Tested natural compounds that showed no effects against $D$. suzukii are also listed. Knowledge gaps that currently hinder a widespread use of natural compounds for controlling D. suzukii are summarized. This review was written following the recommendations outlined by Pautasso (2013).

\subsection{Classification of tested compounds}

The natural compounds tested for controlling D. suzukii can be classified according to their currently established general mode of action:

\section{Compounds}

- that have a repulsive effect on the flies are subsequently referred to as repellents,

- that require physical contact for expressing toxic effects are subsequently referred to as contact toxicants,

- that require oral uptake for expressing toxic effects are subsequently referred to as feeding toxicants,

- that have an activity via the gaseous phase are subsequently referred to as fumigants,

- that reduce the number of eggs laid are subsequently referred to as oviposition repellents and that fatally affect egg development as ovicides.

Some compounds may have multiple modes of action and therefore can belong to several groups. It is also possible that some compounds have effects that are not yet known. Therefore, the present classification represents the current state of knowledge and may be subject to change when new experimental studies provide additional evidence.

\subsection{General considerations on estimated toxicity parameters}

Three toxicity parameters were used to describe the effect of the compounds on D. suzukii.

The dose lethal for $50 \%$ of the exposed individuals, usually called $\mathrm{LD}_{50}$, was used to estimate the level of contact toxicity of test compounds within a certain time (Porteus 2008). LD 50 values are usually given in milligrams of product per kilogram of animal body mass. For small animals like flies, $\mathrm{LD}_{50}$ values are sometimes given as micrograms of product per individual.

The lethal concentration $\left(\mathrm{LC}_{50}\right)$ is the concentration of a substance in air or water that is sufficient to kill $50 \%$ of a sample population within a certain time (Porteus 2008). This parameter was used to measure the fumigant toxicity.

The half-maximally effective concentration, also called $\mathrm{EC}_{50}$, is the concentration at which $50 \%$ of the maximum effect is produced or the concentration of drug at which the drug is half-maximally effective (Lowe and Balis 2012). This parameter was used to measure contact and fumigant toxicities.

Whenever the magnitude of effects of natural compounds on $D$. suzukii had to be estimated from graphs published by other authors, the software tool WebPlotDigitizer version 4.1 was used.

\section{Repellents}

Potential repellents were tested using either T-maze assays, choice trap assays, impregnated cotton wicks or direct airborne repellent assays. The results of repellent tests are summarized by assay in the following paragraphs.

\subsection{T-maze and choice trap assays}

A T-maze assay is based on a T-shaped apparatus: a test odorant is positioned into one arm of the T-maze and a control odorant into the opposite arm. Flies are released into the Tmaze apparatus and after a predetermined experimentation time, the position of flies is recorded and a preference index is calculated (Eq. 1) (Krause Pham and Ray 2015).

Preference index $=\frac{\text { number of flies in test arm-number of flies in control arm }}{\text { number of flies in test arm }+ \text { number of flies in control arm }}$

A trap assay is set up in a closed arena; for a no-choice assay, only one trap is arranged (treatment and control studied separately) and for a choice assay, at least two traps are arranged (treatment and control). Generally, test compounds are applied to a filter paper. Traps can have several designs. To test the effect of pyridine and DEET substitutes, the treated filter paper was placed at the entry of the trap and flies had to crawl over it to reach the lure in the trap. After a predetermined experimentation time, the number of flies in each trap was counted and the preference index was calculated (Eq. 1) (Krause Pham and Ray 2015). To study the repellence of octenol, geosmin and benzaldehyde, the filter paper was arranged in the trap and after a predetermined time, the number of flies in each trap recorded (Renkema et al. 2016; Wallingford et al. 2016b).

Carbon dioxide and pyridine (an animal skin odorant) are known to repel other Drosophila species and therefore, their effect against $D$. suzukii was assessed in a T-maze assay (Krause Pham and Ray 2015). Carbon dioxide concentration (concentration tested $=\mathrm{CT}=0 ; 0.34 ; 0.67$ and $1.34 \% \mathrm{v} / \mathrm{v}$ ) in the air had no repellent effect on $D$. suzukii with a similar preference index for all concentrations tested, whereas $\mathrm{CO}_{2}$ was strongly avoided by $D$. melanogaster. Pyridine $(\mathrm{CT}=1 \%$ $\mathrm{v} / \mathrm{v})$ slightly attracted the flies. A complementary experiment, a two-choice trap assay, was also conducted to study the long- 
term effect of pyridine; $D$. suzukii showed no significant avoidance for this product (Krause Pham and Ray 2015).

Most Drosophila species are repelled by DEET ( $N, N$-diethylmeta-toluamide), a commonly used synthetic insect repellent. The repellent activity of three natural substitutes to DEET (butyl anthranilate (BA), methyl $N, N$-dimethylanthranilate (MDA) and ethyl anthranilate (EA)) $(\mathrm{CT}=1$ and $10 \% \mathrm{v} / \mathrm{v})$ was assessed in a two-choice trap assay and their preference index were estimated. At $1 \%$, the most repellent product was DEET followed by BA, MDA and EA with preference index of $-0.8,-0.6,-0.3$ and 0.05 , respectively. At a concentration of $10 \%$, all the products showed a repellent activity with preference index of -0.6 for DEET and EA, and -0.4 for BA and MDA. Thus, at $1 \%$, only BA had a repellent effect comparable to DEET, while at $10 \%$, all the natural substitutes repelled D. suzukii as DEET (Krause Pham and Ray 2015).

Two-choice trap assays were conducted to ascertain whether octenol, geosmin and benzaldehyde (CT $=0.1 ; 1$ and $10 \%$ in mineral oil v/v) could repel $D$. suzukii from crushed raspberries mixed with yeast. Octenol and geosmin significantly repelled flies at 1 and $10 \%$ but not at a lower concentration. Benzaldehyde did not repel D. suzukii (Wallingford et al. 2016b). Interestingly, octenol and geosmin are known to be produced by necrotrophic fungi, such as Penicillium expansum and Botrytis cinerea on infected grape berries (La Guerche et al. 2005). It may be speculated that flies are repelled by these substances to avoid oviposition into already decaying fruits that may not allow the full development of the larvae.

\subsection{Impregnated cotton wicks}

The repellent effect of the EO of Pelargonium asperum, Mentha $\times$ piperita, Zingiber officinale, Eucalyptus radiata, Cymbopogon winterianus, Lavandula angustifolia, Rosmarinus officinalis, Thymus vulgaris, Thuja occidentalis, Abies balsamea, Picea glauca and Pinus strobus was assessed against D. suzukii (Renkema et al. 2016). A cotton wick was soaked with the EO and transferred into a vial containing fresh raspberry juice. The flies could choose between an untreated cotton wick and the treated one, and their position was recorded after 1, 6 and $24 \mathrm{~h}$. The EO of P. asperum, Z. officinale, Mentha $\times$ piperita, $C$. winterianus, $L$. angustifolia and T. vulgaris significantly repelled the flies for $24 \mathrm{~h}$ while the other tested EO showed a weaker repellent activity (Renkema et al. 2016). In a similar experiment, the repellent effect of the EO of $C$. winterianus, E. radiata, P. asperum, Mentha $\times$ piperita and T. vulgaris, their major compounds (at the rate in the $\mathrm{EO}$ ) and a blend of the major compounds (at the rate in the EO) was assessed against $D$. suzukii (Renkema et al. 2017). The only difference compared with the previous experiment was the use of a blueberry juice as the lure instead of raspberry juice. The flies could choose between an untreated cotton wick and the treated one; their position was recorded after 2, 6 and $24 \mathrm{~h}$.
The most efficient EO were extracted from $C$. winterianus, $P$. asperum and T. vulgaris, which significantly repelled D. suzukii males and females for at least $24 \mathrm{~h}$. Among the major compounds of the EO, only thymol $(\mathrm{CT}=35 \% \mathrm{w} / \mathrm{v})$ showed a significant repellent activity against both sexes until $24 \mathrm{~h}$ when tested individually. Other compounds such as geraniol $(\mathrm{CT}=$ $22 \%)$ and citronellol $(\mathrm{CT}=34 \%)$ significantly repelled males for $24 \mathrm{~h}$ and females for $6 \mathrm{~h}$. The blends made of the major compound of $P$. asperum and T. vulgaris significantly repelled the flies for $24 \mathrm{~h}$. Following these results, three blends of compounds were prepared, one dominated by thymol, one dominated by citronellol and a four-compound blend (thymol, citronellol, menthol and geraniol). Cotton wicks were soaked with the blends and placed between two fresh raspberries. Choice and no-choice assays were conducted. In the choice assay, the thymol blend significantly repelled landing on raspberries for 6 and $24 \mathrm{~h}$ whereas the repellent effect of the citronellol blend was only significant at $24 \mathrm{~h}$. The four-compound blend had no repellent activity. In the no-choice assay, the repellent activity of the thymol blend was the same; it significantly repelled flies from landing on raspberries after 6 and $24 \mathrm{~h}$. The citronellol blend had no repellent activity whereas the four-compound blend had a significant repellent effect only at $24 \mathrm{~h}$ (Renkema et al. 2017).

\subsection{Direct airborne repellent assay}

The direct airborne repellent test is suitable for volatile compounds that could be confounded by responses from the taste system. In a $20-\mathrm{cm}$ length tube, a filter paper is placed at each end, one is treated with the tested compound and the other one with the control. A brass screen prevents contact between flies and the filter paper. Flies are introduced into the tube and after a predetermined experimentation time, their position is recorded $(5 \mathrm{~cm}$ near the treated or the untreated end) (Krause Pham and Ray 2015). Citronellal (CT $=0.1$ and $1 \% \mathrm{v} / \mathrm{v})$, a compound present in numerous EO, is a known repellent so it has been tested against $D$. suzukii. At the lower concentration, citronellal attracted $D$. suzukii and, at $1 \%$, it slightly repelled the flies; thus, this product did not have a significant effect on the behaviour of D. suzukii (Krause Pham and Ray 2015).

\subsection{Conclusion on repellents}

The most promising repellents tested against $D$. suzukii so far were butyl anthranilate, octenol, geosmin, the EO of P. asperum, C. winterianus and T. vulgaris and the EO compound thymol.

\section{Contact toxicants}

Contact toxicity was assessed by different methods. The most frequently used method was direct application of the product 
to the flies with a syringe. This method will subsequently be referred to as topical application and was mainly employed for EO, their major compounds and fungal metabolites. For formulations based on bacteria or entomopathogenic fungi, products were transferred to absorbent materials such as cotton or felt and D. suzukii was exposed to them in a closed arena.

\subsection{Topical application}

The experimental protocol for topical application was described previously (Kim et al. 2016b). The flies were kept in the following environmental conditions to obtain the results summarized below $23-27{ }^{\circ} \mathrm{C}, 60-70 \%$ relative humidity and 16:8 L:D (16-h light, 8-h darkness).

Among the tested substances, the most effective were Leptospermum ericoides and Leptospermum scoparium EO, the polar fraction of those $\mathrm{EO}$ and $L$. scoparium $\mathrm{EO}$ triketone fraction with $\mathrm{LD}_{50}$ values between 0.13 and $0.71 \mu \mathrm{g} /$ fly for males and 0.22 and $1.23 \mu \mathrm{g} / \mathrm{fly}$ for females (Fig. 2, Jang et al. 2017; Kim et al. 2016a, 2016b; Park et al. 2017, 2016). The contact toxicity of the non-polar fraction, made up of sesquiterpene hydrocarbons, was significantly lower than the toxicity of the polar fraction. The triketone fraction as part of the polar fraction was mainly composed of flavesone, isoleptospermone and leptospermone (Park et al. 2017). For Leptospermum ericoides, the polar fraction was 67 -fold more effective than the non-polar fraction for males and 134-fold more effective for females. For Leptospermum scoparium, the factor between the efficiency of the two fractions was 19 for males and 39 for females.
Cypermethrin, a pyrethroid insecticide, was used as a positive control. The contact toxicity of this product was very high with $\mathrm{LD}_{50}$ values ranging from $0.05 \times 10^{-3}$ to $0.13 \times 10^{-3} \mu \mathrm{g} /$ fly for $D$. suzukii males and from $0.06 \times 10^{-3}$ to $0.44 \times 10^{-3} \mu \mathrm{g} /$ fly for females, indicating that this synthetic product has an insecticidal activity approximately $10^{3}$-fold higher than the most efficient of the tested EO or their compounds (Jang et al. 2017; Kim et al. 2016b; Park et al. 2017, 2016).

Other EO had a significant contact toxicity compared to the control at the highest concentration tested but were not promising enough to estimate their $\mathrm{LD}_{50}$, such as the $\mathrm{EO}$ of Leptospermum citratum, Pimenta dioica, Syzygum aromaticum (Park et al. 2017), Hyssopus officinalis, Mentha piperita, Ocimum basilicum, Origanum majorana (Park et al. 2016), Eucalyptus polybractea, Melalenca alternifolia, M. dissitiflora, M. ericifolia, M. viridiflora (Jang et al. 2017), Croton anisatum, Aniba roseodora, Jasminum sambac, Amyris balsamifera, Illicium verum and Styrax tonkiensis (Kim et al. 2016b).

Finally, some EO tested showed no contact toxicity, such as the EO of Lavandula angustifolia, Rosmarinus officinalis, Salvia sclarea, Vitex agnus castus (Park et al. 2016), Eucalyptus globulus, E. radiata, E. smithii, M. leucadendron, M. uncinata (Jang et al. 2017), Rhus taratana, Bursea delpechiana, Boswellia carterii, Chamaecyparia obtusa, Cryptomeria japonica, Cupressus sempervirens, Juniperis communis, Thujopsis dolabrata, Gaultheria fragrantissima, Cedrus atlantica, Pinus sylvestris and Citrus limonum (Kim et al. 2016b).

Fungal metabolites (sparassol, methyl orsellinate and methyl 2,4-dimethoxy-6-methylbenzoate (DMB)) were applied

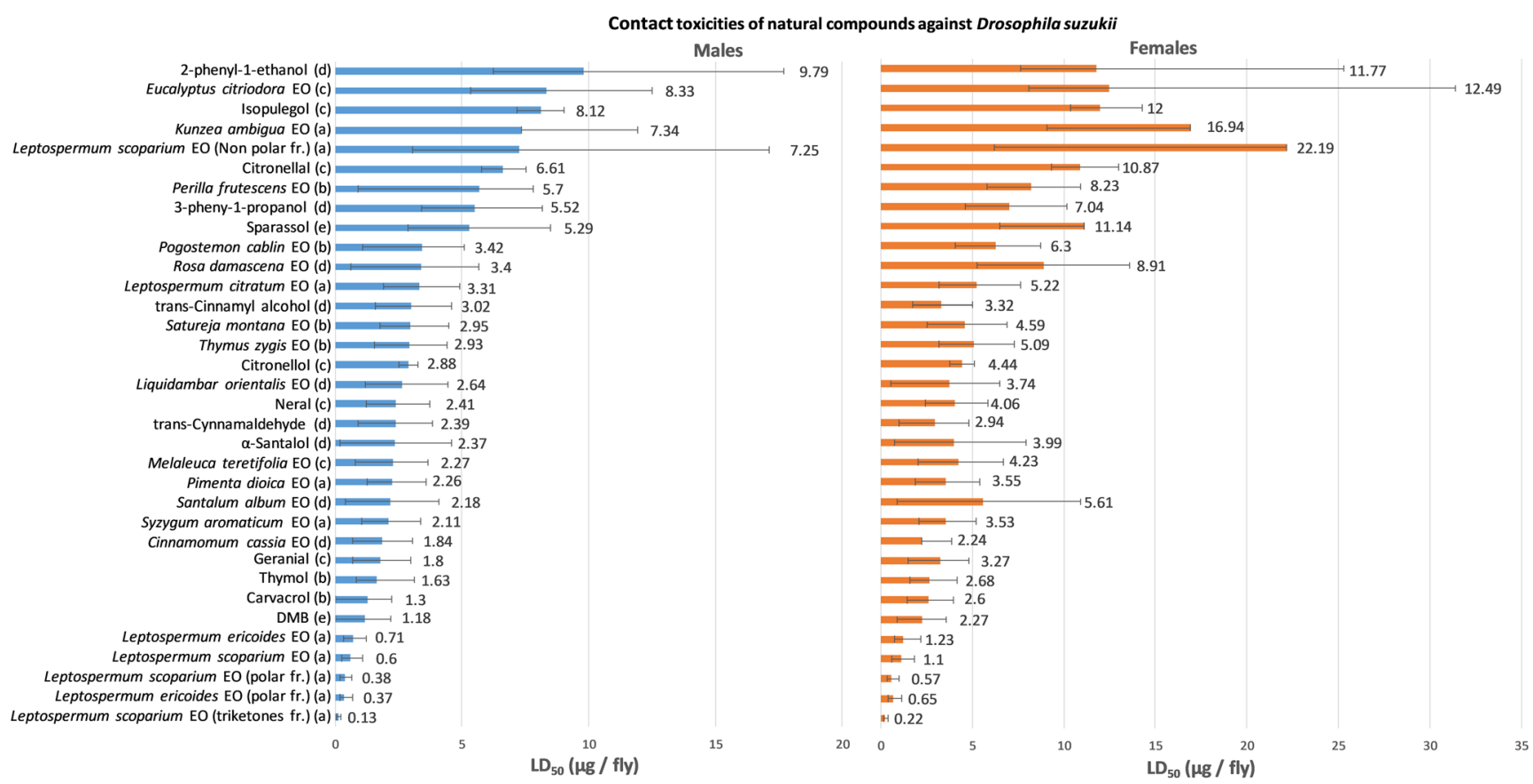

Fig. 2 Reported contact toxicities of natural compounds versus Drosophila suzukii. EO = essential oil; fr. = fraction. Error bars represent $95 \%$ confidence limits. Data were published by (a) Park et al. (2017); (b) Park et al. (2016); (c) Jang et al. (2017); (d) Kim et al. (2016b) and (e) Kim et al. (2016a) 
topically to D. suzukii. Only DMB and sparassol showed an interesting contact toxicity with $\mathrm{LD}_{50}$ of 1.18 and $5.29 \mu \mathrm{g} / \mathrm{fly}$ for males, respectively, and 2.27 and $11.14 \mu \mathrm{g} /$ fly for females, respectively (Fig. 2, Kim et al. 2016a).

Generally, $\mathrm{LD}_{50}$ values were higher for females than for males (Fig. 2, Jang et al. 2017; Kim et al. 2016a, b; Park et al. 2017, 2016). Females tend to be bigger than males (average body length when raised with cherry: females $2.5 \mathrm{~mm}$; males $2.25 \mathrm{~mm}$ or with grapes: females $4.0 \mathrm{~mm}$; males $3.0 \mathrm{~mm}$ (Walsh et al. 2011 adapted from Kanzawa)), but there seems to be no evidence supporting a role of body size for the differential sensitivity at present.

The $\mathrm{EC}_{50}$ was also used to compare the contact toxicity of some EO. All the EOs tested had a significant insecticidal activity, except that of Aleurites moluccana. Persea americana EO, 1,8-cineole and L. latifolia EO showed the highest insecticidal activity with $\mathrm{EC}_{50}$ values of $0.54,0.67$ and $0.69 \%$ of the product when $0.5 \mathrm{ml}$ was applied per fly (Fig. 3, Erland et al. 2015). The EO of Azadirachta indica, Macadamia integrifolia, several cultivars of Lavandula and the compounds $\delta$-carene and linalool showed a weaker contact toxicity (Fig. 3, Erland et al. 2015).

\subsection{Use of impregnated matrices}

In a series of studies, the contact toxicity of selected compounds was assessed with the test compounds being impregnated into a support matrix like a cotton wick or a piece of felt, and the percentage of dead flies counted after predetermined time intervals was used to compare the products.

The entomopathogenic fungus Metarhizium brunneum (strain EAMa 01/58-Su) was tested on a felt cone impregnated with conidia (CT $=3 \times 10^{10}$ conidia). An artificial diet positioned at the bottom of the cone attracted the flies, such that the flies had to crawl over the inoculated felt when trying to reach the feed. After 5 days of exposure, this fungal strain had a significant contact toxicity compared to the control, inducing $62.2 \%$ and $57.7 \%$ of mortality with a black and a red felt, respectively. Treated adults had an average survival time (AST) of 3.6 days. The horizontal transmission of the entomopathogenic fungus was also studied. Flies (males and females separately) were transferred into the device described above for $24 \mathrm{~h}$. Then, the following combinations were tested for 7 days: infected males with noninfected females, infected females with non-infected males, both infected or both non-infected. When males were infected, $72 \%$ of them died within 7 days and induced a mortality of $24 \%$ in untreated females with an AST of 6.3 days. When females were infected, $84 \%$ of them died and induced the mortality of $48 \%$ in untreated males with an AST of 5.6 days. When both were infected, mortality was $88 \%$ and $84 \%$ for males and females, respectively. When both were untreated, only $8 \%$ of males and $7 \%$ of females died during the experiment. The horizontal transmission of the entomopathogenic fungus induced significant mortality when only males, only females or both are infected compared to uninfected. In the same experiment, the sub-lethal effect of the entomopathogenic fungus was studied by counting the number of emerged pupae. When males were infected, the number of pupae was similar that of when males and females were healthy with a mean of 22 pupae. When either both sexes or only females were infected, the number of pupae was significantly lower with a mean of four pupae, corresponding to a reduction of $84.7 \%$ (Yousef et al. 2018).

D. suzukii was exposed to commercial formulations of Bacillus thuringiensis on cotton wicks. None of the tested products induced mortality in the tested populations suggesting their lack of insecticidal activity against D. suzukii (Biganski et al. 2018).

\section{Contact toxicities of natural compounds against Drosophila suzukii}

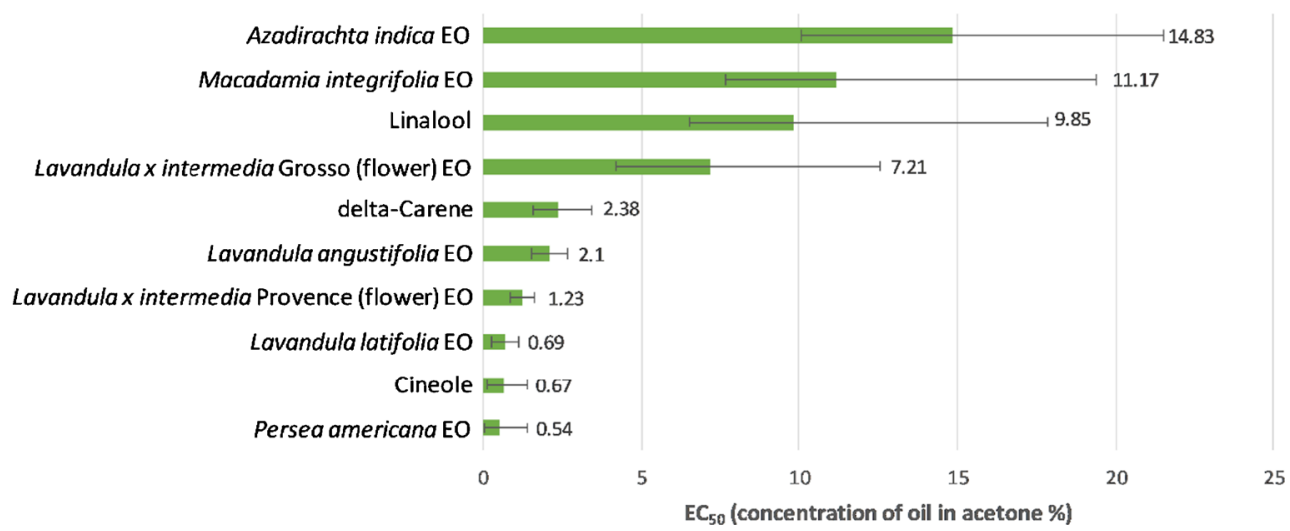

Fig. 3 Reported contact toxicities of natural compounds versus Drosophila suzukii. EO = essential oil. Error bars represent 95\% confidence limits. Data were published by Erland et al. (2015) 


\subsection{Conclusion on contact toxicants}

By topical application, the EO of L. scoparium and L. ericoides applied fully or partially (e.g. only the polar fraction) were the most promising. The entomopathogenic fungus Metarhizium brunneum had an interesting insecticidal effect with medium rates of horizontal transmission and sub-lethal effects.

\section{Ingestion or contact toxicants}

Some experimental set-ups do not allow a clear distinction between the effects of ingestion and contact. These cases will be discussed together in the following paragraphs.

\subsection{Feeding and development on a supplemented medium}

The insecticidal effect of a chitinase extracted from the latex of Euphorbia characias was tested against D. suzukii larvae. The chitinase was mixed with an artificial diet $(\mathrm{CT}=0.005 \%$ and $0.025 \% \mathrm{w} / \mathrm{v}$ ) in which larvae could feed and develop. At $0.005 \%$, the chitinase caused $93 \%$ mortality among the larvae and only $2 \%$ developed into adults. At $0.025 \%$, larvae neither survived nor pupated after 10 days of treatment. Both tested chitinase concentrations induced a significant mortality in the brood compared to the control. They were as efficient as the synthetic insecticide diflubenzuron (Dimilin ${ }^{\circledR}, \mathrm{CT}=2 \% \mathrm{v} / \mathrm{v}$ ) which had a slower activity inducing $20 \%$ of larval mortality and $100 \%$ of pupal mortality (Martos et al. 2017).

The insecticidal activity of a crude extract of Metarhizium brunneum (EAMb 09/01-su), an entomopathogenic fungus, was assessed on D. suzukii adults. The crude extract was mixed with an artificial diet and had a significant contact/ feeding toxicity with $88.8 \%$ of mortality after $48 \mathrm{~h}$ compared to $3.3 \%$ in the control. Exposure periods of between 6 and $9 \mathrm{~h}$ were needed to achieve $50 \%$ mortality. The crude extract was also tested in a lure-and-kill trap-device: in this case too, the crude extract showed a significant insecticidal activity with $61.6 \%$ of dead flies compared to $3.3 \%$ in the control (Yousef et al. 2018).

The toxicity of erythritol (Truvia ${ }^{\circledR}, \mathrm{CT}=0$ to $2 \mathrm{M}$ ) to $D$. suzukii was assessed by allowing flies to feed on supplemented artificial media. Within a 4-day exposure period, the $\mathrm{LC}_{50}$ of erythritol was $1.15 \mathrm{M}$ and the $\mathrm{LC}_{90}$ was $1.82 \mathrm{M}$. When larvae were feeding on the same treated artificial diet media, they died before pupation at an erythritol concentration in the media of $0.5 \mathrm{M}$ (Sampson et al. 2017b). As erythritol may be cost-prohibitive, the efficiency of lower cost derivatives has been investigated against $D$. suzukii (Sampson et al. 2019). Flies were allowed to feed on a medium supplemented with pentaerythritol, dipentaerythritol, tripentaerythritol, erythritol $(\mathrm{CT}=0.25,0.5$ or $1 \mathrm{M})$ or water as a negative control for 7 days. Then, the media were kept two more weeks for counting pupae and emerging adults. Only erythritol and pentaerythritol induced a significant adult mortality with respective rates of $90 \%$ and $100 \%$ at $1 \mathrm{M}$. Concerning adult emergence, erythritol had a significant effect with a reduction of $90 \%$ at a concentration of $0.25 \mathrm{M}$. At $1 \mathrm{M}$, pentaerythritol and dipentaerythritol induced a reduction of $60 \%$ and $80 \%$ of adult emergence, respectively. However, under laboratory conditions, only erythritol was able to prevent the increase of the population whereas pentaerythritol and dipentaerythritol only slowed it down (Sampson et al. 2019). The insecticidal activity of erythritol may be explained by a dual mode of action: (i) starvation when it is the sole food source and (ii) desiccation induced by cytolysis. Indeed, erythritol is not metabolized and diffuses through the midgut membrane to reach the hemolymph where it induces a quick increase of osmotic pressure in the fly's body, which can be lethal. The higher efficiency of erythritol and pentaerythritol compared to dipentaerythritol and tripentaerythritol was linked to their smaller molecule size and their reduced number of hydroxyl groups which induce a greater osmotic pressure (Sampson et al. 2019).

The insecticidal activity of Bacillus thuringiensis-commercialized formulations against $D$. suzukii larvae and pupae was studied. These products were mixed with an artificial medium on which larvae feed and develop. The percentage of mortality of larvae and pupae was very low and not significantly different from the control for all the products tested so far, suggesting no significant toxicity against $D$. suzukii larvae (Biganski et al. 2018).

\subsection{Feeding on and contact with a soaked cotton wick}

The ethanolic seed extracts (ESE) of the Annona species were offered to the flies on an impregnated cotton wick as sole food source for $24 \mathrm{~h}$ (CT $=2000 \mathrm{mg} / \mathrm{l}$ of water). Five days after the exposure period, the mortality of the flies was assessed. ESE of A. muricata did not induce a significant mortality rate compared to the control whereas the ESE of A. sylvatica and A. mucosa did, with $35 \%$ and $94 \%$ of fly mortality, respectively. The ESE of A. mucosa showed an insecticidal effect comparable to the synthetic insecticide spinetoram (Delegate $\left.250 \mathrm{WG}^{\mathrm{TM}}, \mathrm{CT}=300 \mathrm{mg} / \mathrm{l}\right)$. The ingestion toxicity of the ESE of A. mucosa was influenced by the exposure time: the $\mathrm{LC}_{50}$ was $1666.13 \mathrm{mg} / \mathrm{l}$ for an exposure of $12 \mathrm{~h}$ and decreased to $500.43 \mathrm{mg} / 1$ for $120 \mathrm{~h}$ (Bernardi et al. 2017).

The insecticidal activity of non-nutritive sugars against D. suzukii was assessed with impregnated cotton wick assays. The sugars tested were erythritol, erythrose, xylitol, mannitol, sorbitol and nutritive sugar sucrose (positive control) (CT = $0.05 ; 0.1 ; 0.5$ and $1 \mathrm{M}$ ). Water was used as a negative control. Irrespective of the concentration, erythrose and erythritol resulted 
in the highest rates of mortality without survivors after 7 days of experiment. Among all tested non-nutritive sugars, only these two had a significant ingestion toxicity with results comparable to the negative control (Choi et al. 2017). The most promising non-nutritive sugar, erythritol, was tested mixed with sucrose to stimulate feeding and thus increase its ingestion and possibly its efficiency (Choi et al. 2017; Cowles et al. 2015). Two experimental set-ups were arranged: in the first one, erythritol (CT = $0.5 ; 1$ and $2 \mathrm{M})$ and sucrose $(\mathrm{CT}=0.5$ and $1 \mathrm{M})$ were offered separately each in one tube in different combinations of concentration. Only one combination $(0.5 \mathrm{M}$ erythritol in one tube and water in the other) showed a significant mortality rate compared to the positive control ( $0.5 \mathrm{M}$ sucrose and water) with no survival after 6 days. The other combinations showed almost no fly mortality. In the second set up, erythritol $(\mathrm{CT}=0.5 ; 1$ and $2 \mathrm{M})$ and sucrose $(\mathrm{CT}=0.5$ and $1 \mathrm{M})$ were mixed in the same tube. In this experiment, several combinations showed a significant ingestion toxicity compared to the control but the most promising was the combination $1 \mathrm{M}$ sucrose/2 $\mathrm{M}$ erythritol, followed by $0.5 \mathrm{M}$ sucrose/2 $\mathrm{M}$ erythritol and $1 \mathrm{M}$ sucrose/ $1 \mathrm{M}$ erythritol with no survival after 3, 4 and 5 days, respectively (Choi et al. 2017). A similar procedure was used in another study: all the combinations tested showed a significant insecticidal activity against $D$. suzukii with the most promising combinations $1 \mathrm{M}$ sucrose $/ 2 \mathrm{M}$ erythritol and $0.5 \mathrm{M}$ sucrose/2 $\mathrm{M}$ erythritol resulting in $100 \%$ of mortality after 3 and 4 days of feeding, respectively (Tang et al. 2017). To better reflect field conditions where alternative feed sources are often available, the insecticidal activities of erythritol and its combination with sucrose were studied in the presence of wounded blueberries (Choi et al. 2019). Ten flies were allowed to feed on a cotton wick soaked with $0.5 \mathrm{M}$ erythritol or $0.5 \mathrm{M}$ sucrose or $2 \mathrm{M}$ erythritol combined with $0.5 \mathrm{M}$ sucrose in the presence of 0,20 or $60 \%$ of wounded blueberries after a starvation period of $24 \mathrm{~h}$. The survival was checked daily for 7 days. Erythritol alone induced comparable mortality rates (around 60\%) regardless of the presence of wounded berries while sucrose alone had no insecticidal activity. The combination of erythritol and sucrose was the most effective treatment with $100 \%$ of mortality for berries with intact cuticle, $85 \%$ of mortality when $20 \%$ of the berries were wounded and $87 \%$ of mortality when $60 \%$ of the berries were wounded (Choi et al. 2019). The insecticidal activity of the combination of erythritol and sucrose against $D$. suzukii had already been reported by Choi et al. (2017) and Tang et al. (2017); however, this experiment showed that it had a significant lethal effect also in the presence of naturally occurring sugars provided by wounded berries (Choi et al. 2019).

The contact or ingestion toxicity of the EO of $P$. asperum, Mentha $\times$ piperita, $Z$. officinale, E. radiata, $C$. winterianus, L. angustifolia, R. officinalis, T. vulgaris, T. occidentalis, A. balsamea, P. glauca and P. strobus was tested against D. suzukii flies. A choice bioassay was set up where flies could choose between a vial containing raspberry juice and a treated
$(\mathrm{CT}=15 \mathrm{~g} / \mathrm{l})$ or untreated cotton wick. None of the EO tested showed a significant insecticidal effect (Renkema et al. 2016).

The toxicity by contact or ingestion of blends made of EO compounds was assessed. One was mainly composed of thymol, one of citronellol and the last one was composed by thymol, citronellol, menthol and geraniol. These blends were applied to a cotton wick and arranged near a raspberry. The mortality of flies was assessed in choice tests ( 1 treatment vs control) or no-choice tests. In the first case, no difference was observed in mortality rates between all the treatments. In the no-choice assay, the thymol dominated blend showed a mortality significantly higher than the control, the citronellol and the four-compound blends (Renkema et al. 2017).

\subsection{Feeding on or contact with treated fruits}

Blueberries were rolled in powdered sulphur (Bago d'Ouro $\AA$, $\mathrm{CT}=2.6 \pm 0.3 \mathrm{mg} /$ fruit) or sprayed with kaolin (Surround $\AA$, $\mathrm{CT}=5 \% \mathrm{w} / \mathrm{v})$. Blueberries were available to the flies for $48 \mathrm{~h}$ and direct mortality was assessed. Afterwards, blueberries were removed and residual mortality was recorded after seven additional days. Powdered sulphur induced a significant direct mortality with $23.1 \%$ of dead flies (5.3\% for females and $40 \%$ for males). Seven days after exposure, the residual mortality rates were $11.1 \%$ for females and $73.4 \%$ for males. Hence, powdered sulphur had a significant insecticidal activity primarily on D. suzukii males. Kaolin expressed no toxicity with mortality rates comparable to the ones observed in the control (Pérez-Guerrero and Molina 2016).

Kaolin (Surround $\AA, C T=2 \% \mathrm{w} / \mathrm{v}$ ), clinoptilolite (Klinospray®, $\mathrm{CT}=2 \% \mathrm{w} / \mathrm{v}$ ) alone or mixed with the adjuvant Heliosol $(\mathrm{CT}=0.5 \% \mathrm{v} / \mathrm{v})$, calcium carbonate $(\mathrm{CT}=1.7 \mathrm{~g} / \mathrm{l})$, calcium hydroxide (Nekapur®, $\mathrm{CT}=1.7 \mathrm{~g} / \mathrm{l}$ ) and diatomaceous earth (Pflanzen-Aktivator ${ }^{\circledR}$ P2032, CT $=0.34 \mathrm{~g} / \mathrm{l}$ ) were tested against D. suzukii. Blueberries were sprayed with the products using a spinning table spray booth. A white coating was observed on the fruit after spraying. None of the tested substances showed insecticidal activity against $D$. suzukii after an exposure period of $24 \mathrm{~h}$ (Strack et al. 2017). In the studies of PérezGuerrero and Molina (2016) and Strack et al. (2017), kaolin showed no insecticidal activity against $D$. suzukii.

To assess the insecticidal activity of methyl benzoate (CT = $0.1 ; 0.5 ; 1$ and $5 \% \mathrm{v} / \mathrm{v}$ ) on D. suzukii, blueberries were dipped in the solution. The toxicity of this product was concentration-dependant. After $48 \mathrm{~h}$ of exposure, a concentration of $0.1 \%$ of methyl benzoate had no significant effect. For the higher concentrations, methyl benzoate had a significant insecticidal effect with $50 \%$ fly mortality at $0.5 \%$ and almost no survival at concentrations of $1 \%$ and 5\% (Feng and Zhang 2017).

Since erythritol mixed with sucrose showed an interesting insecticidal activity against $D$. suzukii when added to an artificial media, a complementary test was made in a greenhouse with treated fruits. In a tent, a blueberry bush and 
commercialized blueberries arranged in a hanging basket were sprayed with the solutions of erythritol mixed with sucrose $(\mathrm{CT}=0.5 \mathrm{M}$ sucrose $/ 2 \mathrm{M}$ erythritol or $1 \mathrm{M}$ sucrose $/ 2 \mathrm{M}$ erythritol). All the combinations were significantly different from the controls with the quickest mortality observed for the combination $0.5 \mathrm{M}$ sucrose $/ 2 \mathrm{M}$ erythritol followed by $0.5 \mathrm{M}$ erythritol and $1 \mathrm{M}$ sucrose/2 $\mathrm{M}$ erythritol (Tang et al. 2017).

In another experiment, blackberries were dipped in erythritol (Truvia $\AA, C T=2 \mathrm{M}$ ) alone or mixed with a synthetic growth regulator (erythritol $\mathrm{CT}=1 \mathrm{M}+$ lufenuron $\mathrm{CT}=$ $0.5 \mathrm{ppm}$ ) before being offered to D. suzukii for oviposition. Erythritol alone induced $93 \%$ of male mortality and $83 \%$ of female mortality, while mixed with lufenuron, its insecticidal activity decreased slightly with respective mortality rates of $82.2 \%$ and $78.3 \%$. These mortality rates were not statistically different from those observed with the negative control which was only water $(72.6 \%$ of male mortality and $69.2 \%$ of female mortality) (Sampson et al. 2017b). However, a positive control seemed to be missing in the latter study.

\subsection{Conclusion on ingestion or contact toxicants}

Added to an artificial medium, the entomopathogenic fungus M. brunneum and the chitinase extracted from E. characias showed high toxicity against adults and larvae of D. suzukii, respectively. Erythritol mixed with sucrose had a high insecticidal activity as food source on a cotton wick or as fruit treatment. Powdered sulphur and methyl benzoate showed an interesting toxicity when used as a fruit treatment.

\section{Fumigants}

Fumigants are volatile poisonous chemicals released into the air or injected into the soil to kill pests. Fumigant toxicity has often been evaluated using impregnated paper allowing the release of the product into the air of a closed experimental chamber (Erland et al. 2015; Kim et al. 2016b). The experimental set-up protected the flies from coming into contact with the impregnated paper thanks to a sieve or a mesh. The products tested were mainly EO, some of their major compounds and fungal metabolites.

Against $D$. suzukii males, perilla aldehyde, geranial and neral had the highest insecticidal activity with $\mathrm{LC}_{50}$ values of $0.99,1.36$ and $1.52 \mathrm{mg} / \mathrm{l}$ air, respectively (Fig. 4). Against the females, the most efficient products were perilla aldehyde, geranial and menthol with $\mathrm{LC}_{50}$ values of $1.15,1.88$ and $1.94 \mathrm{mg} / 1$ air, respectively (Fig. 4). These compounds are the major components of the EO of Perilla frutescens, Melaleuca teretifolia and Mentha $\times$ piperita (Jang et al. 2017; Kim et al. 2016b; Park et al. 2017, 2016)

Dichlorvos, an organophosphorus insecticide, was used as a positive control. The fumigant toxicity of this product was very high with $\mathrm{LC}_{50}$ values ranging from $0.24 \times 10^{-3}$ to $0.86 \times 10^{-3} \mathrm{mg} / 1$ air for $D$. suzukii males and from $0.36 \times$ $10^{-3}$ to $1.08 \times 10^{-3} \mathrm{mg} / 1$ air for females. The insecticidal activity of this synthetic product is approximately $10^{4}$ times higher than that of the EOs or their compounds (Jang et al. 2017; Kim et al. 2016b; Park et al. 2017, 2016).

Other EOs had a significant fumigant toxicity compared to the control at the highest concentration tested but were not promising enough to estimate their $\mathrm{LD}_{50}$ such as the EO H. officinalis, L. angustifolia, O. basilicum, O. majorana, S. sclarea, S. montana, T. zygis (Park et al. 2016), M. alternifolia, M. dissitiflora, M. ericifolia (Jang et al. 2017), T. dolabrata, A. roseodora and J. sambac (Kim et al. 2016b).

Some EO tested showed no fumigant toxicity such as the EO of L. ericoides, L. scoparium, K. ambigua, P. dioica, S. aromaticum (Park et al. 2017), P. cablin, R. officinalis, $V$. agnus castus (Park et al. 2016), E. globulus, E. polybractea, E. radiata, E. smithii, M. leucadendron, M. uncinata, $M$. viridiflora (Jang et al. 2017), L. occidentalis, $R$. taratana, B. carterii, C. obtusa, C. japonica, C. sempervirens, $J$. communis, $C$. atlantica, $P$. sylvestris, $R$. damascena, A. balsamifera, C. limonum, S. album and S. tonkiensis (Kim et al. 2016b).

As observed for contact toxicants with $\mathrm{LD}_{50}$, females had higher $\mathrm{LC}_{50}$ values than males (Fig. 4, Jang et al. 2017; Kim et al. 2016b; Park et al. 2017, 2016).

Product efficiencies were also compared based on $\mathrm{EC}_{50}$ values for the EO of $P$. americana, A. moluccana, M. integrifolia, A. indica, several cultivars of Lavandula and the compounds 1,8-cineole, $\delta$-carene and linalool. Among all these products, only the EO of Lavandula cultivars and the isolated compounds has a significant fumigant toxicity. Linalool, L. latifolia EO and leaf L. intermedia (cultivar: Provence) EO were the most effective products against D. suzukii with $\mathrm{EC}_{50}$ values of $1.85,3.79$ and $5.68 \mu \mathrm{loil} / 1$ air, respectively (Fig. 5, Erland et al. 2015).

The fumigant toxicity of the fungal metabolites (sparassol, methyl orsellinate and DMB) was very low with mortality rates below 4\% (Kim et al. 2016a).

\section{Fumigants and contact toxicants}

Several EO and their major compounds expressed both contact and fumigant toxicities. For the EO of M. teretifolia, L. citratum, P. frutescens and E. citriodora as well as their major compounds geranial, neral, citronellal and isopulegol, average $\mathrm{LD}_{50}$ values for contact toxicity and average $\mathrm{LC}_{50}$ values for fumigant toxicity were related (Fig. 6) with females being slightly less sensitive than males, particularly when the contact toxicity of citronellal, isopulegol and EO of Eucalyptus citriodora was concerned (Fig. 6). Approximately $2.1 \mu \mathrm{g}$ and $2.5 \mu \mathrm{g}$ of natural product per male and female were needed in contact toxicity assays to obtain the 


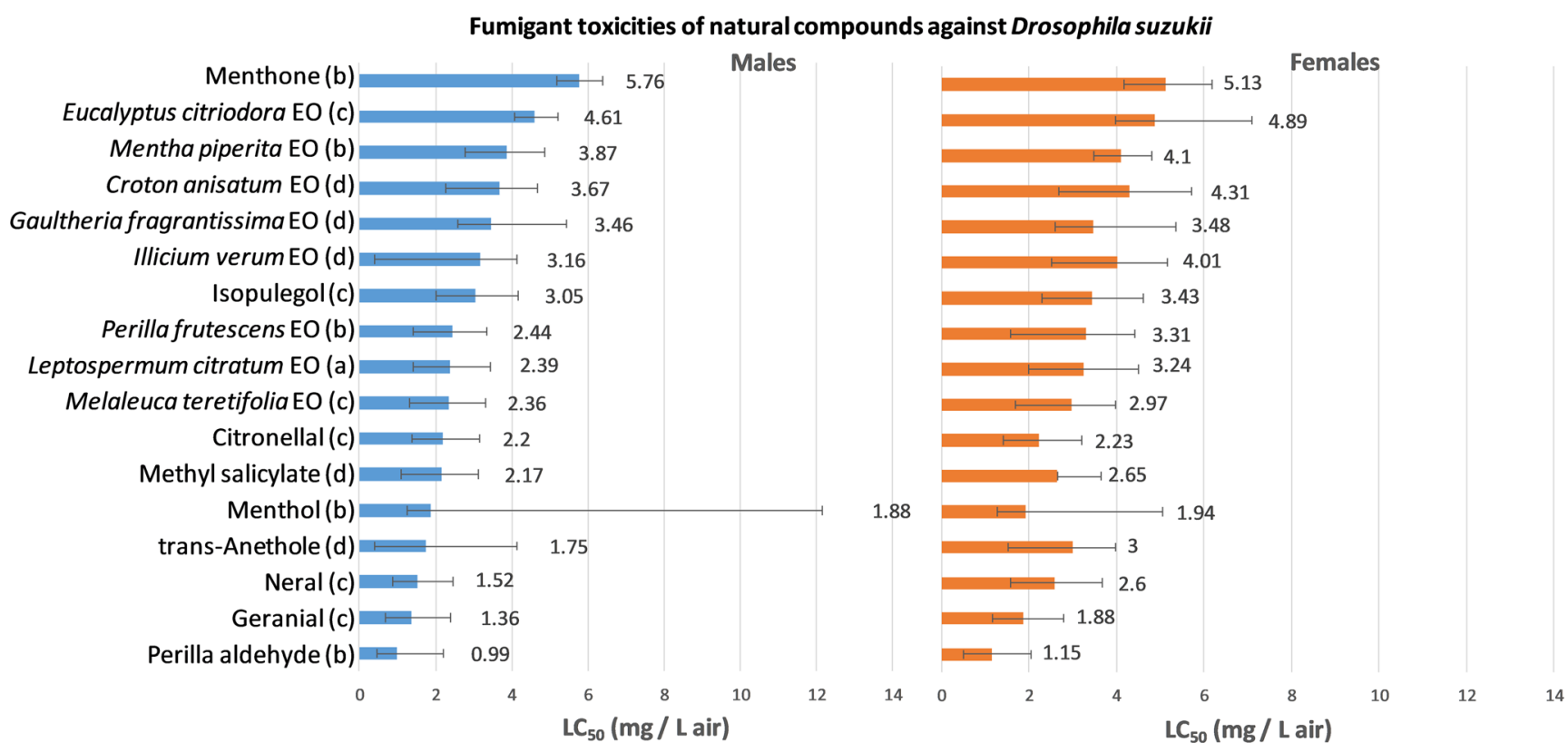

Fig. 4 Reported fumigant toxicities of natural compounds versus Drosophila suzukii. EO = essential oil. Error bars represent $95 \%$ confidence limits. Data were published by (a) Park et al. (2017); (b) Park et al. (2016); (c) Jang et al. (2017) and (d) Kim et al. (2016b)

effect of $1 \mathrm{mg}$ natural product per litre of air in fumigant toxicity assays, respectively (Fig. 6, Jang et al. 2017; Kim et al. 2016b; Park et al. 2017, 2016). For the EO of L. latifolia, L. angustifolia and flowers of Lavandula $\times$ intermedia Provence and Grosso and the compounds linalool, delta-carene and 1,8-cineole (Erland et al. 2015), there was no correlation between contact and fumigant toxicities (data not shown).

\section{Oviposition repellents and ovicides}

Effects of tested compounds on oviposition were quantified by counting (i) the number of eggs deposited in fruit or (ii) the number of larvae or pupae that hatched or the number of adults that emerged from fruit. Numerous products such as EO, powders or bacterial formulations were tested. The most frequently used method was to treat fruits (such as raspberries, strawberries, blackberries or blueberries) directly. Other methods are usually based on the arrangement of the products close to the fruits to test potential repellent effects on the flies.

As fruits are not equally attractive for oviposition (Bellamy et al. 2013), the efficiency of the products will be described depending on the fruit that was used in the studies published so far.

\subsection{Raspberries}

The effect on the oviposition of EO blends was tested. One blend was mainly composed of thymol, another one of citronellol and the last one composed of thymol, citronellol, menthol

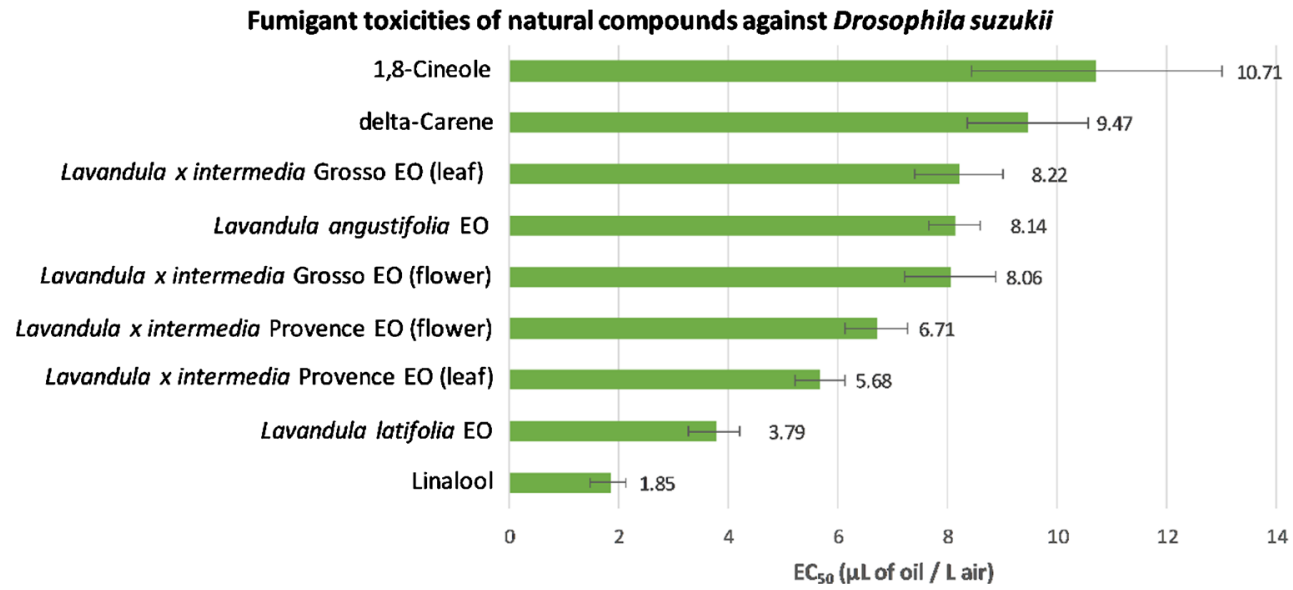

Fig. 5 Reported fumigant toxicities of natural compounds versus Drosophila suzukii. EO=essential oil. Error bars represent $95 \%$ confidence limits. Data were published by Erland et al. (2015) 
Fig. 6 Comparison of contact and fumigant toxicities of geranial, neral, citronellal, Melaleuca teretifolia EO, Leptospermum citratum EO, Perilla frutescens $\mathrm{EO}$, isopulegol and Eucalyptus citriodora $\mathrm{EO}$ in D. suzukii males and females. Males are represented by up triangles and females by down triangles. Data were published by Jang et al. (2016), Kim et al. (2016b) and Park et al. (2016, 2017). Error bars represent the $95 \%$ confidence limits. Correlations were significant at $P=0.0211$ for males and non-significant for females at $P=0.1191$

\section{$\mathrm{LD}_{50}(\mu \mathrm{g} / \mathrm{fly})$, contact toxicity}

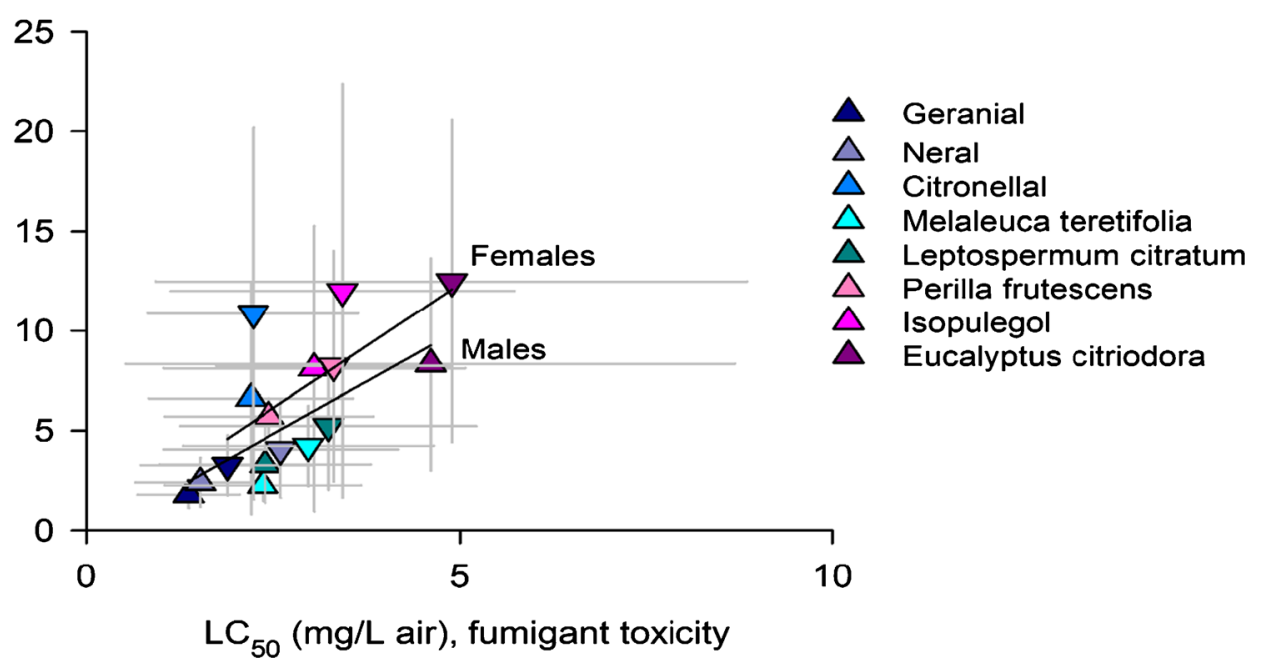

and geraniol. Two raspberries were placed on either side of a treated cotton wick in no-choice and choice tests. In the nochoice experiment, the thymol blend was the only one with a significant effect on the larval infestation. In choice assays, all the blends induced a significant reduction $(68-76 \%)$ of the number of larvae per berry compared to the fruits with an untreated wick. In the repellent part, all the blends showed a variable repellent effect on flies' landings on raspberries. It is possible that the reduction in larvae observed and subsequently of eggs laid is due to the reduction in landings (Renkema et al. 2017).

The effect of edible coatings (carnauba-based protective coating (PrimaFresh® 45), carnauba wax organically modified with kaolin clay (Raynox® Apple Sunburn Protectant) and calcium carbonate shade product (REFLECTIONSTM)) on oviposition and brood survival has been studied in raspberries. Fruit were coated (CT $=1: 40$ to 1:1 product:water ratio) and offered for oviposition for $4 \mathrm{~h}$ in a cage. At the end of the oviposition time, eggs were counted and fruit were kept 21 additional days for monitoring adult emergence. Only Raynox ${ }^{\circledR}$ and PrimaFresh $®$ significantly reduced the number of eggs laid in raspberries with a reduction of $62.3 \%$ and $65 \%$ respectively at $1: 10$ and almost $70 \%$ at $1: 2$. These products also significantly reduced the number of eggs reaching the pupal stage with no pupae from concentrations of 1:5 for Raynox and of 1:2 for PrimaFresh® (Swoboda-Bhattarai and Burrack 2014).

The effect of octenol and geosmin on D. suzukii oviposition was studied on several occasions. In a laboratory choice test, octenol and geosmin (CT $=1$ and $10 \%$ in water $\mathrm{v} / \mathrm{v})$ were applied to cotton wicks and placed close to raspberries. Eggs laid in the berries were recorded. A significant avoidance for oviposition in fruits placed near a treated cotton wick was observed with reductions of $35 \%$ for octenol at $1 \%, 56 \%$ for octenol at $10 \%$ and $52 \%$ for geosmin irrespective of concentration (Wallingford et al. 2017). A similar experiment was conducted in a greenhouse. In a sleeve cage, raspberries were arranged around a cotton wick treated with octenol $(\mathrm{CT}=1$, 10 and $50 \%$ in mineral oil v/v). Octenol at 50\% resulted in a significant reduction $(49.8 \%)$ of the number of eggs laid in berries compared to the control (Wallingford et al. 2016a). In the field, octenol and geosmin were tested in various set-ups. Octenol $(\mathrm{CT}=50 \%$ in mineral oil $\mathrm{v} / \mathrm{v})$, geosmin $(\mathrm{CT}=50 \%$ in distilled water $\mathrm{v} / \mathrm{v}$ ) and a mix of both compounds was arranged in odour dispensers near ripening fruit clusters in an insecticide-free planting of raspberry. The number of eggs laid in fruits was counted and raspberries were kept until adult emergence for identification. Octenol and its combination with geosmin resulted in significantly fewer eggs compared with the control with reductions of $40 \%$ and $43 \%$, respectively, while geosmin alone did not protect the fruit (Wallingford et al. 2017). In a comparable experiment (Wallingford et al. $2016 \mathrm{~b})$, octenol $(\mathrm{CT}=50 \%$ in mineral oil v/v) led to a significant reduction $(41.5 \%)$ of the eggs laid in the berries near an octenol odour dispenser compared to fruits near a control dispenser. Octenol also induced a significant reduction $(47.6 \%)$ of adult emergence. In another experiment, octenol $(\mathrm{CT}=$ $20 \% \mathrm{v} / \mathrm{v}$ ) was added to a specific pheromone and lure application technology (SPLAT) from ISCA technologies (Wallingford et al. 2016a). The system was mounted into the canopy at fruit height in a raspberry orchard. Ripe fruit were harvested and transferred to rearing containers until adult emergence for species identification and counting. Four days after establishing the system in the orchard, the treated plots showed significantly fewer offspring/g of fruit with reductions ranging from 28.8 to $49.5 \%$ compared to the control plots. However, 7 days after the deployment of the system in the planting, no differences in infestation levels were observed between treated and untreated plots any more (Wallingford et al. 2016a), suggesting a short-term efficacy of the latter method. Octenol was studied in the comparison of "push", "pull" and "push-pull" strategies. These strategies aimed to 
modify the geographical localization of the pest thanks to an aversive stimuli to "push" it or an attractive lure to "pull" it away from the crop (Wallingford et al. 2018). In the laboratory, experiments were carried out in a cage where octenol $(\mathrm{CT}=25 \%$ in mineral oil $\mathrm{v} / \mathrm{v}$ ) was used as a repellent in the "push" and "push-pull" strategies and was applied to a cotton wick arranged near the raspberries. An attractive trap was used in the "pull" and "push-pull" strategies. Compared to the control, all the strategies show a significant reduction in the number of eggs per fruit. The "push" and the "pull" strategies had similar results with respective reductions of $66.2 \%$ and $69.6 \%$ whereas the "push-pull" strategy was the most effective with $87.6 \%$ fewer eggs per fruit. In the field, octenol (CT $=50 \%$ in mineral oil $\mathrm{v} / \mathrm{v}$ ) was arranged in an odour dispenser. The "push" and "push-pull" strategies showed a significant reduction in the number of eggs laid in fruits, $56.7 \%$ and $57.4 \%$ respectively. On the contrary, the "pull" system had an increase of $44.1 \%$ of infestation compared to the control. However, the latter result may be due to the use of a sticky card in the trap instead of a drowning solution, which may have diminished the efficacy of the "pull" system (Wallingford et al. 2018). Octenol had a repellent effect against $D$. suzukii.

\subsection{Strawberries}

The effect of ESE from Annona species on oviposition was also assessed. In a no-choice experiment, ripe strawberries were dipped in a solution of ESE (CT = $2000 \mathrm{mg}$ of ESE/l) and deposited eggs were counted after $24 \mathrm{~h}$. All the ESE tested showed a significant reduction in the number of eggs laid compared to untreated fruits ( $45 \%$ for A. mucosa, <35\% for A. sylvatica and A. muricata) and a higher oviposition repellence than spinetoram (Delegate $250 \mathrm{WG}^{\mathrm{TM}}$, CT $=300 \mathrm{mg} / \mathrm{l}$ ), which induced a reduction of $26 \%$ (Bernardi et al. 2017).

The effect of three edible coatings on $D$. suzukii infestation was studied in a strawberry field. PrimaFresh ${ }^{\circ} 45$, Raynox $®$ and REFLECTIONSTM $(\mathrm{CT}=1: 10$ product:water ratio) were sprayed and 7 days later, ripe strawberries were harvested and monitored for 21 days for adult emergence and identification. None of these products reduced the number of adults that emerged from strawberries compared to untreated fruits (Swoboda-Bhattarai and Burrack 2014).

\subsection{Blackberries}

Blackberries were dipped in erythritol (Truvia ${ }^{\circ}, \mathrm{CT}=2 \mathrm{M}$ ) alone or mixed with a synthetic growth regulator (erythritol $\mathrm{CT}=1 \mathrm{M} /$ lufenuron $\mathrm{CT}=0.5 \mathrm{ppm}$ ) before being offered to D. suzukii for oviposition. These formulations showed a number of eggs laid that was comparable to the control but induced a significant reduction in the progeny survival. Erythritol alone led to an $88.9 \%$ reduction in survival and mixed with lufenuron, 99\% mortality was observed. Eggs failed to hatch or larvae died shortly after hatching (Sampson et al. 2017b). In a field trial, erythritol (Truvia Baking Blend ${ }^{\circledR}, \mathrm{CT}=0.5 \mathrm{M}$ ), lufenuron $(\mathrm{CT}=10 \mathrm{ppm})$ and the combination of both were applied on blackberry plants once a week for 3 weeks before fruit harvest. None of the treatments had a discernible effect on the number of eggs laid but they reduced larval infestation by 71 to $79 \%$ (Sampson et al. 2017a).

\subsection{Blueberries}

Blueberries were used in many studies even though according to Bellamy et al. (2013), they are not among the most attractive fruit for D. suzukii.

For products with a powder formulation, such as powdered sulphur (Bago d'Ouro $®)$, blueberries were rolled in the powder. In a no-choice and a choice assay (CT $=2.6 \pm 0.3 \mathrm{mg}$ / fruit), powdered sulphur significantly reduced the number of eggs laid in blueberries by $76 \%$ and $97 \%$, respectively (PérezGuerrero and Molina 2016).

Kaolin (Surround®, CT $=5 \% \mathrm{w} / \mathrm{v}$ ) was sprayed on blueberries. This product had no effect on oviposition in no-choice and choice assays with a number of eggs laid in treated fruit similar to the control (Pérez-Guerrero and Molina 2016). In another study (Strack et al. 2017), kaolin (Surround®, CT = $2 \% \mathrm{w} / \mathrm{v}$ ) sprayed on blueberries showed a significant reduction in the number of eggs deposited $(86.4 \%)$ which was comparable to the effect of spinosad (Audienz®, $\mathrm{CT}=0.025 \%$ $\mathrm{v} / \mathrm{v}$ ), a synthetic insecticide that quickly killed the flies inducing a reduction in the number of eggs laid by $95.2 \%$. These studies showed contrasting effects of kaolin on oviposition that may be explained by the difference in the experimentation (variety and number of blueberries, number of flies, time of exposure, and method of spraying). Further studies are needed to clarify the effect of kaolin on D. suzukii oviposition.

Strack et al. (2017) also tested other powdered coatings, such as calcium carbonate $(\mathrm{CT}=1.7 \mathrm{~g} / \mathrm{l})$, clinoptilolite (Klinospray®, $\mathrm{CT}=2 \% \mathrm{w} / \mathrm{v}$ ) alone or mixed with the adjuvant Heliosol $(\mathrm{CT}=0.5 \% \mathrm{v} / \mathrm{v})$, calcium hydroxide (also called lime, Nekapur®, $\mathrm{CT}=1.7 \mathrm{~g} / \mathrm{l}$ ) and diatomaceous earth (Pflanzen-Aktivator ${ }^{\circledR}$ P2032, CT = $0.34 \mathrm{~g} / \mathrm{l}$ ). Calcium carbonate and the two formulations of clinoptilolite significantly reduced the number of eggs deposited, respectively by $80.5 \%$, $74.2 \%, 75.6 \%$. Only calcium hydroxide and diatomaceous earth had a non-significant effect on oviposition (Strack et al. 2017).

An experiment under semi field conditions with blueberry plants in pots under a mesh cage indicated that lime (Nekapur $2 ®, \mathrm{CT}=1.8 \mathrm{~g} / \mathrm{l})$ reduced the number of eggs laid in fruit. The product was sprayed once a week for 4 weeks. Each week, the treated plants showed a lower infestation than control plants but only the fourth week of treatment showed a significant difference with a reduction of $98.5 \%$ in the number of eggs 
laid (Dorsaz et al. 2017b). This study showed different results from the ones described by Strack et al. (2017) this may be due to the difference in the experimental conditions (blueberries variety, fly, time of exposure, treatment) or this can be linked to the way of working of lime with a slow effect not measurable in the study of Strack et al. (2017).

The effect of edible coatings (PrimaFresh ${ }^{\circledR} 45$, Raynox ${ }^{\circledR}$ and REFLECTIONSTM) on oviposition and brood survival has been studied in blueberries. Fruit were coated $(\mathrm{CT}=$ 1:40 to $1: 1$ product:water ratio) and offered for oviposition for $4 \mathrm{~h}$ in a cage. At the end of the oviposition time, eggs were counted and fruit were kept 21 additional days for monitoring adult emergence. Only Raynox ${ }^{\circledR}$ and REFLECTIONSTM significantly reduced the number of eggs laid in blueberries with reductions of $65.5 \%$ and $73 \%$ respectively at a $1: 2$ product:water ratio. However, these products had no effect on the number of eggs reaching the pupal stage and the number of pupae that emerged as adults inducing that they should be used before the presence of D. suzukii in the crop (Swoboda-Bhattarai and Burrack 2014).

Erythritol, a non-nutritive sugar, was assessed for its oviposition deterrence. Flies were allowed to lay eggs in untreated blueberries for 7 days while feeding on a cotton stud soaked with various erythritol solutions. Erythritol solutions alone $(\mathrm{CT}=0.5$ to $2.0 \mathrm{M})$ or mixed with sucrose solutions $(\mathrm{CT}=$ 0.5 or $1 \mathrm{M}$ ) led to a significant decrease in the number of eggs compared to the control ( $0.5 \mathrm{M}$ sucrose). The most efficient formulations for avoiding oviposition were $0.5 \mathrm{M}$ sucrose/ $2 \mathrm{M}$ erythritol and $1 \mathrm{M}$ sucrose/ $1 \mathrm{M}$ erythritol with less than two eggs counted per blueberry. The result suggested that erythritol reduced female fecundity (Tang et al. 2017). In a similar experiment, flies were allowed to lay eggs in untreated blueberries for $48 \mathrm{~h}$ while feeding on a cotton stud soaked with erythritol $(\mathrm{CT}=0.5 \mathrm{M})$, sucrose $(\mathrm{CT}=0.5 \mathrm{M})$ or their combination (2 M erythritol $+0.5 \mathrm{M}$ sucrose). Erythritol alone or mixed with sucrose induced a reduction of $43 \%$ of the number of eggs laid compared to sucrose (Choi et al. 2019) supporting previous results by Tang et al. (2017). To better understand the reason of the reduced fecundity, female flies were dissected to count the number of ovarian eggs. When studying the combination of eggs laid and ovarian eggs, females fed with erythritol alone had 12.4 eggs, on erythritol and sucrose 16.5 eggs and on sucrose alone 19.3 eggs. It was speculated that this reduction was caused by a deteriorated nutritional status of the flies since erythritol is not metabolized. Females fed with the combination of erythritol and sucrose laid fewer eggs but had a higher number ovarian eggs compared to sucrose fed flies showing a reduction of the oviposition activity (Choi et al. 2019). In a field trial, erythritol (Truvia Baking Blend $囚, C T=0.5 \mathrm{M}$ ), lufenuron $(\mathrm{CT}=10 \mathrm{ppm})$ and the combination of both compounds was applied on blueberry bushes once a week for 3 weeks before fruit harvest (Sampson et al. 2017a). None of the treatments had a discernible effect on the number of eggs deposited but they reduced larval infestation. Indeed, erythritol induced $100 \%$ of mortality for the first instar of larvae and $43 \%$ for the second instar. In untreated blueberries, for each larvae that survived, 8 eggs had to be laid. When blueberries were treated with erythritol, 54 eggs were necessary and with the combination of erythritol and lufenuron, 59 eggs were needed to obtain one surviving larva. None of the treatments prevented oviposition and crop damage completely but they induced an average reduction of $75 \%$ of larval infestation and thus the population decreases (Sampson et al. 2017a). The lower cost derivative of erythritol, pentaerythritol, showed an interesting efficiency against $D$. suzukii in laboratory trials and was therefore selected for testing in a blueberry field (Sampson et al. 2019). Weekly treatments were applied using a backpack sprayer (untreated control or $0.5 \mathrm{M}$ of erythritol in water + soap or $0.5 \mathrm{M}$ of pentaerythritol in water + soap or $0.25 \mathrm{M}$ of erythritol $+0.25 \mathrm{M}$ of pentaerythritol in water + soap) for the last 3 weeks prior to harvest. A similar effect of erythritol and pentaerythritol (both of them tested at $0.5 \mathrm{M}$ ) was observed with a significant reduction of the number of eggs laid (64\%) as well as the number of hatching larvae (94\%). The combination of erythritol and pentaerythritol induced a higher reduction in the number of eggs deposited ( $82 \%$ of reduction) and a similar control of larvae (94\% of reduction) (Sampson et al. 2019).

To learn if butyl anthranilate (BA) $(\mathrm{CT}=1 ; 2.5 ; 5$ and $10 \%$ $\mathrm{v} / \mathrm{v}$ ), a DEET substitute, has an effect on oviposition, blueberries were painted with the product or its solvent and offered to the flies for 1 week. Then, the number of eggs, larvae and pupae was counted. A dose-dependent effect was found with a reduction in the offspring emergence ranging from $12 \%$ at $1 \%$ of BA; $47 \%$ at a concentration of $2.5 \%$; $77 \%$ with a $5 \%$ concentration and $97 \%$ at $10 \%$ (Krause Pham and Ray 2015).

The effect on oviposition of the EO of $P$. americana, A. moluccana, L. angustifolia, L. latifolia, Lavandula $\times$ intermedia (cultivars: Grosso and Provence) (leaf or flower), M. integrifolia and A. indica and their major compound cineole, $\delta$-carene and linalool $(\mathrm{CT}=1 ; 5 ; 10$ and $20 \% \mathrm{v} / \mathrm{v})$ was assessed by dipping blueberries and counting the number of oviposition marks after a 48-h period of oviposition. None of these EO had a significant effect on oviposition (Erland et al. 2015).

The effect on oviposition of Bacillus thuringiensis formulations was tested by dipping blueberries in the solutions in a choice experiment. The mean number of larvae per fruit was not significantly different between treated and untreated fruits, suggesting that these products had no effect on oviposition at the concentrations and conditions used so far (Biganski et al. 2018).

To assess the ovicidal effect of a product, blueberries were offered for oviposition until eggs were laid. Subsequently, half of the fruit was dipped in a solution of the test product. The number of offspring was compared between treated and untreated blueberries. This method was used to assess the ovicidal effect of methyl benzoate $(\mathrm{CT}=1 \% \mathrm{v} / \mathrm{v})$ (Feng and Zhang 2017) and lime (Nekapur $2 \AA, C T=1.8 \mathrm{~g} / \mathrm{l})($ Dorsaz 
et al. 2017a). Methyl benzoate showed a significant toxicity against $D$. suzukii offspring as no larvae or pupae developed in treated blueberries (Feng and Zhang 2017). Lime also induced a significant reduction in adult emergence: $24 \%$ of eggs developed further to adults in treated fruit compared with $57 \%$ in untreated fruits (Dorsaz et al. 2017a).

\subsection{Conclusion concerning the effect on oviposition}

Since just a few larvae can render the crop useless, the efficacy of treatments against $D$. suzukii oviposition needs to be high. Treatments that resulted in more than $90 \%$ of efficiency were powdered sulphur (Bago d'Ouro ${ }^{\circledR}, \mathrm{CT}=2.6 \mathrm{mg} /$ fruit) applied directly on fruit, erythritol $(\mathrm{CT}=1 \mathrm{M})+\operatorname{sucrose}(\mathrm{CT}=1 \mathrm{M})$, erythritol $(\mathrm{CT}=2 \mathrm{M})+$ sucrose $(\mathrm{CT}=0.5 \mathrm{M})$ and erythritol $(\mathrm{CT}=0.5 \mathrm{M})$ in the feed. These products were primarily applied to blueberries. More testing is needed because the efficiency of the products varies depending on the fruit and because the vast majority of experiments were no-choice tests that do not reflect field conditions. Even though D. suzukii can inflict heavy damage on red vine cultivars, studies on the prevention of oviposition in this economically important crop are scarce.

\section{Limitations of using natural compounds for controlling $D$. suzukii}

This review shows that several structurally diverse natural compounds had promising repellent or insecticidal effects on D. suzukii. However, some points currently limiting a widespread use were also noted.

\subsection{High concentrations needed for efficacy}

To obtain a similar level of protection as provided by synthetic insecticides, most of the natural compounds tested need to be approximately 1000 times more concentrated. For example, the most effective contact toxicant showed that the triketone fraction of the $\mathrm{EO}$ of Lesptospermum scoparium had $\mathrm{LD}_{50}$ of $0.13 \mu \mathrm{g} /$ fly for males and $0.22 \mu \mathrm{g} /$ fly for females whereas the $\mathrm{LD}_{50}$ of the synthetic insecticide cypermethrin is below $0.06 \times 10^{-3} \mu \mathrm{g} / \mathrm{fly}$ irrespective of the sex (Park et al. 2017). These high concentrations imply financial and technical challenges. The rather high requirement of natural compounds can easily render their use non-profitable as discussed by Sampson et al. (2017a, b) for erythritol. Since the production of the plants or fungi that contain the interesting natural compounds is also associated with (ecological) costs, life cycle analyses are needed to understand whether producing the most effective natural compounds on a large scale in plants or fungi is ecologically and economically sustainable. The risk of phytotoxicity and thus crop damage usually increases with concentration, a point that has rarely been addressed and tested in the studies published so far, but that may easily render effective compounds useless for crop protection.

\subsection{Transfer to and deposition of natural compounds on crops}

More efficient methods for transferring and depositing natural compounds onto the crops than those used in the papers summarized here need to be established and optimized without efficacy losses during the process. For example, for spray applications, natural compounds need to be diluted with a non-toxic, cheap and generally available carrier liquid (usually water). However, lipophilic compounds such as oils are not mixable with water. Appropriate formulations must be developed; or, carrier liquids other than water are required. Technologies are needed for depositing powders or waxy compounds in a homogenous manner on fruit surfaces. Concerning ingestion toxicants, erythritol and sucrose combinations are very sticky, limiting uptake by the flies (Tang et al. 2017). The physicochemical properties of the compounds need to be adapted for instance by developing formulations for widespread use with commercially available equipment.

\subsection{Competition for use}

Many of the tested compounds are "generally considered as safe" (GRAS) and are already used in food, pharmaceuticals or cosmetics, like EO (flavour and fragrance in food and beverages, perfumes, personal care products) and erythritol (calorie-reduced food, candies, toothpaste). Competition for the resource is likely, and again, life cycle analyses that consider this aspect are needed to better understand the contribution that the natural compounds can make when taking economic boundary conditions into consideration.

\subsection{Occasional lack of specificity}

Some of the tested products have multiple activities beyond the insecticidal one against $D$. suzukii. For example, the triketones fraction of the Leptospermum $\mathrm{EO}$ had an insecticidal contact activity against $D$. suzukii but triketones and more particularly leptospermone has an herbicidal activity and its synthetic derivatives belong to the $\beta$-triketone class of herbicides. The mode of action of these herbicides is the inhibition of the enzyme p-Hydroxyphenylpyruvate dioxygenase (Dayan et al. 2007). Flavesone, another triketone, is the active component of Flavocide ${ }^{\mathrm{TM}}$, an insecticide used against various pests (Miller and Peters 2017). Erythritol inhibits tomato and corn seed germination and decreases tomato growth at insecticidal doses showing a potential herbicidal effect (Scanga et al. 2018). A lack of specificity with regard to biological activity implies a huge group of non-target organisms 
that are likely to be exposed to additional risks. This aspect needs to be investigated thoroughly before widespread use, particularly of those natural compounds that have a rather broad spectrum of biological activity.

\subsection{Lack of knowledge of the (molecular) modes of action}

The mode of action of the products and their target site(s) have rarely been investigated and hardly ever determined. The inhibitory activity of EO and their major compounds on acetylcholinesterase (AChE) and/or glutathione-S-transferase (GST) has been assessed (Jang et al. 2017; Kim et al. 2016b; Park et al. 2016). Only a few compounds show a potent inhibition activity against $\mathrm{AChE}$, such as perilla aldehyde, thymol, carvacrol and trans-cinnamaldehyde whereas neral and geranial inhibit GST. However, these inhibitory activities do not unambiguously indicate that these enzymes are the factual and only target site of the tested products. More generally, the exact mode of action of EO is unknown but a likely target site is the octopaminergic system (Enan 2001). The mode of action of the coatings discussed in the present paper is also largely unknown. They may modify the fruit surface characteristics such as the $\mathrm{pH}$ or the colour and render it unsuitable for oviposition (Swoboda-Bhattarai and Burrack 2014), but evidence supporting these assumptions is scarce, so far.

The efficacy of many products summarized in the present paper has not been confirmed under field conditions, so far. Repellent products not applied directly to the fruits but to a cotton wick or in a dispenser may act differently in the field (dilution in the air or by rain, decomposition by heat) reducing their efficiency. Contact toxicants may be sprayed but the probability that they directly reach a fly in the field is low. Ingestion products may express a very variable performance because of the competition with natural non-toxic food sources. Fumigants are most effective in closed compartment, not under open field conditions. Organic compounds may be quickly colonized and metabolized by microorganisms. More information on the effects of environmental and biotic factors on the performance of the natural compounds is needed.

\subsection{Waiting time until effects unfold}

The period between the application of the treatment and its effects can be critical. Actually, because $D$. suzukii females lay between 7 and 48 eggs per day from the second day of their adult life, and have a short generation time (Cini et al. 2012), it is necessary to kill or repel them before oviposition or to kill the eggs once they have been laid and before they develop to larvae. For example, following the exposure to the entomopathogenic fungus Metarhizium brunneum, D. suzukii adults had an average survival time of 3.6 days (Yousef et al. 2018), which is enough for the infestation of several fruits and may allow the survival or even increase of the population.

As $D$. suzukii finds a shelter in wild hosts near crops, it is necessary that the products keep an insecticidal effect to avoid a quick re-infestation of the crop and to increase the interval between the treatments.

Acknowledgements We thank the Institut Viti-Vinicole (IVV) of Luxembourg for financially supporting the project BioViM, the European Union for supporting the project Clim4Vitis (grant agreement No 810176), Mareike Schulz (IVV) for helpful discussions and Lindsey Auguin for language editing.

\section{Compliance with ethical standards}

Conflict of interest The authors declare that they have no conflict of interest.

\section{References}

Asplen MK, Anfora G, Biondi A, Choi D-S, Chu D, Daane KM, Gibert P, Gutierrez AP, Hoelmer KA, Hutchison WD, Isaacs R, Jiang Z-L, Kárpáti Z, Kimura MT, Pascual M, Philips CR, Plantamp C, Ponti L, Vétek G, Vogt H, Walton VM, Yu Y, Zappalà L, Desneux N (2015) Invasion biology of spotted wing Drosophila (Drosophila suzukii): a global perspective and future priorities. J Pest Sci 88:469-494. https://doi.org/10.1007/s10340-015-0681-z

Bellamy DE, Sisterson MS, Walse SS (2013) Quantifying host potentials: indexing postharvest fresh fruits for spotted wing Drosophila, Drosophila suzukii. PLoS One 8:e61227. https://doi.org/10.1371/ journal.pone.0061227

Bernardi D, Ribeiro L, Andreazza F, Neitzke C, Oliveira EE, Botton M, Nava DE, Vendramim JD (2017) Potential use of Annona by products to control Drosophila suzukii and toxicity to its parasitoid Trichopria anastrephae. Ind Crop Prod 110:30-35. https://doi.org/ 10.1016/j.indcrop.2017.09.004

Biganski S, Jehle JA, Kleespies RG (2018) Bacillus thuringiensis serovar. israelensis has no effect on Drosophila suzukii Matsumura. J Appl Entomol 142:33-36. https://doi.org/10.1111/jen.12415

Choi M-Y, Tang SB, Ahn S-J, Amarasekare KG, Shearer P, Lee JC (2017) Effect of non-nutritive sugars to decrease the survivorship of spotted wing drosophila, Drosophila suzukii. J Insect Physiol 99:86-94. https://doi.org/10.1016/j.jinsphys.2017.04.001

Choi M-Y, Lucas H, Sagili R, Cha DH, Lee JC (2019) Effect of eythritol on Drosophila suzukii (Diptera: Drosophilidae) in the presence of naturally-occurring sugar sources, and on the survival of Apis mellifera (Hymenoptera: Apidae). J Econ Entomol 112:981-985. https://doi.org/10.1093/jee/toy362

Cini A, Ioriatti C, Anfora G (2012) A review of the invasion of Drosophila suzukii in Europe and a draft research agenda for integrated pest management. Bull Insectol 65:149-160

Cowles RS, Rodriguez-Saona C, Holdcraft R, Loeb GM, Elsensohn JE, Hesler SP (2015) Sucrose improves insecticide activity against Drosophila suzukii (Diptera: Drosophilidae). J Econ Entomol 108: 640-653. https://doi.org/10.1093/jee/tou100

Dayan FE, Duke SO, Sauldubois A, Singh N, McCurdy C, Cantrell C (2007) p-Hydroxyphenylpyruvate dioxygenase is a herbicidal target site for $\beta$-triketones from Leptospermum scoparium. Phytochemistry 68:2004-2014. https://doi.org/10.1016/j. phytochem.2007.01.026 
Dorsaz M, Fischer S, Baroffio C-A (2017a) Lutter contre Drosophila suzukii (Matsumura) sans insecticides: est-ce une utopie? Presented at the Association Française de Protection des Plantes 6ème conférence sur les moyens alternatifs de protection pour une production intégrée, Lille

Dorsaz M, Kuonen F, Baroffio C-A (2017b)Current spotted wing drosophila IPM tactics and their practical implementation in berry crops in Switzerland. Acta Hortic 827-832. https://doi.org/10.17660/ ActaHortic.2017.1156.122

Enan E (2001) Insecticidal activity of essential oils: octopaminergic sites of action. Comp Biochem Physiol Part C Toxicol Pharmacol 130: 325-337. https://doi.org/10.1016/S1532-0456(01)00255-1

Erland LAE, Rheault MR, Mahmoud SS (2015) Insecticidal and oviposition deterrent effects of essential oils and their constituents against the invasive pest Drosophila suzukii (Matsumura) (Diptera: Drosophilidae). Crop Prot 78:20-26. https://doi.org/10.1016/j. cropro.2015.08.013

Feng Y, Zhang A (2017) A floral fragrance, methyl benzoate, is Zn efficient green pesticide. Sci Rep 7. https://doi.org/10.1038/srep42168

Hamby KA, Bellamy DE, Chiu JC, Lee JC, Walton VM, Wiman NG, York RM, Biondi A (2016) Biotic and abiotic factors impacting development, behavior, phenology, and reproductive biology of Drosophila suzukii. J Pest Sci 89:605-619. https://doi.org/10.1007/ s10340-016-0756-5

Haye T, Girod P, Cuthbertson AGS, Wang XG, Daane KM, Hoelmer KA, Baroffio C, Zhang JP, Desneux N (2016) Current SWD IPM tactics and their practical implementation in fruit crops across different regions around the world. J Pest Sci 89:643-651. https://doi.org/ 10.1007/s10340-016-0737-8

Jang M, Kim J, Yoon KA, Lee SH, Park CG (2017) Biological activity of Myrtaceae plant essential oils and their major components against Drosophila suzukii (Diptera: Drosophilidae). Pest Manag Sci 73: 404-409. https://doi.org/10.1002/ps.4430

Kim J, Jang M, Lee K-T, Yoon KA, Park CG (2016a) Insecticidal and enzyme inhibitory activities of sparassol and its analogues against Drosophila suzukii. J Agric Food Chem 64:5479-5483. https://doi. org/10.1021/acs.jafc.6b01528

Kim J, Jang M, Shin E, Kim J, Lee SH, Park CG (2016b) Fumigant and contact toxicity of 22 wooden essential oils and their major components against Drosophila suzukii (Diptera: Drosophilidae). Pestic Biochem Physiol 133:35-43. https://doi.org/10.1016/j.pestbp. 2016.03.007

Krause Pham C, Ray A (2015) Conservation of olfactory avoidance in Drosophila species and identification of repellents for Drosophila suzukii. Sci Rep 5. https://doi.org/10.1038/srep11527

La Guerche S, Chamont S, Blancard D, Dubourdieu D, Darriet P (2005) Origin of (-)-geosmin on grapes: on the complementary action of two fungi, Botrytis Cinerea and Penicillium Expansum. Antonie Van Leeuwenhoek 88:131-139. https://doi.org/10.1007/s10482005-3872-4

Langille AB, Arteca EM, Newman JA (2017) The impacts of climate change on the abundance and distribution of the spotted wing Drosophila (Drosophila suzukii) in the United States and Canada. PeerJ 5:e3192. https://doi.org/10.7717/peerj.3192

Lowe EM, Balis FM (2012) Chapter 18: dose-effect and concentrationeffect analysis. In: Principles of Clinical Pharmacology. pp. 289300

Martos S, Spanò D, Agustí N, Poschenrieder C, Pintus F, Moles L, Medda R (2017) A chitinase from Euphorbia characias latex is a novel and powerful plant-based pesticide against Drosophila suzukii. Ann Appl Biol 171:252-263. https://doi.org/10.1111/aab.12369

Miller, P., Peters, B., 2017. Flavesone: a novel insecticide for the control of urban pests. Presented at the ninth international conference on urban pests, Birmingham, pp. 245-251

Park CG, Jang M, Yoon KA, Kim J (2016) Insecticidal and acetylcholinesterase inhibitory activities of Lamiaceae plant essential oils and their major components against Drosophila suzukii (Diptera: Drosophilidae). Ind Crop Prod 89:507-513. https://doi.org/10. 1016/j.indcrop.2016.06.008

Park C, Jang M, Shin E, Kim J (2017) Myrtaceae plant essential oils and their $\beta$-triketone components as insecticides against Drosophila suzukii. Molecules 22:1050. https://doi.org/10.3390/ molecules22071050

Pautasso M (2013) Ten simple rules for writing a literature review. PLoS Comput Biol 9:e1003149. https://doi.org/10.1371/journal.pcbi. 1003149

Pérez-Guerrero S, Molina JM (2016) Short communication: laboratory approach to the use of sulphur and kaolin as preventive control against Drosophila suzukii. Span. J Agric Res 14:e10SC01. https:// doi.org/10.5424/sjar/2016142-8592

Porteus A (2008) Dictionary of environmental science and technology. Dictionnary Environ Sci Technol

Renkema JM, Wright D, Buitenhuis R, Hallett RH (2016) Plant essential oils and potassium metabisulfite as repellents for Drosophila suzukii (Diptera: Drosophilidae). Sci Rep 6. https://doi.org/10.1038/ srep21432

Renkema J, Buitenhuis R, Hallett R (2017) Reduced Drosophila suzukii infestation in berries using deterrent compounds and laminate polymer flakes. Insects 8:117-133. https://doi.org/10.3390/ insects 8040117

Sampson BJ, Marshall DA, Smith BJ, Stringer SJ, Werle CT, Magee DJ, Adamczyk JJ (2017a) Erythritol and lufenuron detrimentally alter age structure of wild Drosophila suzukii (Diptera: Drosophilidae) populations in blueberry and blackberry. J Econ Entomol 110: 530-534. https://doi.org/10.1093/jee/tow307

Sampson BJ, Werle CT, Stringer SJ, Adamczyk JJ (2017b) Ingestible insecticides for spotted wing Drosophila control: a polyol, erythritol, and an insect growth regulator, Lufenuron. J Appl Entomol 141:8 18. https://doi.org/10.1111/jen. 12350

Sampson BJ, Easson MW, Stringer SJ, Werle CT, Magee D, Adamczyk JJ (2019) Laboratory and field assessments of erythritol derivatives on the survival, reproductive rate, and control of Drosophila suzukii (Diptera: Drosophilidae). J Econ Entomol 112:173-180. https:// doi.org/10.1093/jee/toy 274

Scanga SE, Hasanspahič B, Zvorničanin E, Samardžić Koženjić J, Rahme AK, Shinn-Thomas JH (2018) Erythritol, at insecticidal doses, has harmful effects on two common agricultural crop plants. PLoS One 13:e0192749. https://doi.org/10.1371/journal.pone.0192749

Schultz M, Molitor DD (2016) Die Kirschessigfliege - eine neue Herausforderung für den Luxemburger Weinbau. Lëtzebuerger Beien-Ztg 127:354-359

Shawer R, Tonina L, Tirello P, Duso C, Mori N (2018) Laboratory and field trials to identify effective chemical control strategies for integrated management of Drosophila suzukii in European cherry orchards. Crop Prot 103:73-80. https://doi.org/10.1016/j.cropro.2017. 09.010

Strack T, Cahenzli F, Daniel C (2017) Kaolin, lime and rock to control Drosophila suzukii. Presented at the Ökologschen Landbau weiterdenken: Verantwortung übernhemen, Vertrauen stärken

Swoboda-Bhattarai KA, Burrack HJ (2014) Influence of edible fruit coatings on Drosophila suzukii (Matsumura) (Diptera: Drosophilidae) oviposition and development. Int J Pest Manag 60:279-286. https:// doi.org/10.1080/09670874.2014.971453

Tang SB, Lee JC, Jung JK, Choi M-Y (2017) Effect of erythritol formulation on the mortality, fecundity and physiological excretion in Drosophila suzukii. J Insect Physiol 101:178-184. https://doi.org/ 10.1016/j.jinsphys.2017.07.015

Wallingford A, Connelly HL, Dore Brind'Amour G, Boucher MT, MafraNeto A, Loeb GM (2016a) Field evaluation of an oviposition deterrent for management of spotted-wing Drosophila, Drosophila suzukii, and potential nontarget effects. J Econ Entomol 109:17791784. https://doi.org/10.1093/jee/tow116 
Wallingford A, Hesler SP, Cha DH, Loeb GM (2016b) Behavioral response of spotted-wing drosophila, Drosophila suzukii Matsumura, to aversive odors and a potential oviposition deterrent in the field: Drosophila suzukii deterrents. Pest Manag Sci 72:701-706. https:// doi.org/10.1002/ps.4040

Wallingford A, Cha DH, Linn CE, Wolfin MS, Loeb GM (2017) Robust manipulations of pest insect behavior using repellents and practical application for integrated pest management. Environ Entomol 46: 1041-1050. https://doi.org/10.1093/ee/nvx125

Wallingford A, Cha DH, Loeb GM (2018) Evaluating a push-pull strategy for management of Drosophila suzukii Matsumura in red raspberry: push-pull for $D$. suzukii management. Pest Manag Sci 74: 120-125. https://doi.org/10.1002/ps.4666
Walsh DB, Bolda MP, Goodhue RE, Dreves AJ, Lee J, Bruck DJ, Walton VM, O’Neal SD, Zalom FG (2011) Drosophila suzukii (Diptera: Drosophilidae): invasive pest of ripening soft fruit expanding its geographic range and damage potential. J Integr Pest Manag 2: G1-G7. https://doi.org/10.1603/IPM10010

Yousef M, Aranda-Valera E, Quesada-Moraga E (2018) Lure-and-infect and lure-and-kill devices based on Metarhizium brunneum for spotted wing Drosophila control. J Pest Sci 91:227-235. https://doi.org/ 10.1007/s10340-017-0874-8

Publisher's note Springer Nature remains neutral with regard to jurisdictional claims in published maps and institutional affiliations. 